\title{
A Comparison of Pharmacotherapy and Combined Pharmacotherapy with Transcranial Direct Current Stimuli action (tDCS) in Reduction of Impulsivity in Patients with Bipolar Disorder Type I: A Randomized and Parallel Clinical Trial
}

\section{Parnaz Mardani}

Zanjan University of Medical Sciences

Mohsen Dadashi

Zanjan University of Medical Sciences

\section{Hossein Javdani}

Ghazvin University of Medical Sciences: Qazvin University of Medical Sciences

Ahmad Zolghadriha

Zanjan University of Medical Sciences

Elnaz Mousavi ( $\nabla$ elnaz48.mousavi@gmail.com )

Shahid Beheshti university of medical sciences

\section{Research}

Keywords: Bipolar Disorder, Impulsivity, Transcranial Direct Current Stimuli, Medication

Posted Date: May 4th, 2021

DOI: https://doi.org/10.21203/rs.3.rs-438254/v1

License: @ (i) This work is licensed under a Creative Commons Attribution 4.0 International License. Read Full License 


\section{Abstract}

Objective

Bipolar disorder is one of the most destructive psychiatric disorders that leads to many psycho-social problems in patients, besides, the presence of impulsivity doubles this dysfunction and imposes irreparable consequences on the patient. So, in this study we aimed to comparison of pharmacotherapy and combined pharmacotherapy with Transcranial Direct Current Stimuli action (tDCS) in reduction of impulsivity in patients with bipolar disorder type I.

Method

The present study was a clinical trial with pre-test, post-test and a 3-month follow-up period. A sample of 30 patients with bipolar disorder type I was selected by purposive sampling and randomly assigned to two groups (pharmacotherapy and combined pharmacotherapy with tDCS). The tDCS protocol was performed for 10 sessions of 20 minutes for the combined group [F3 F4]. Barret Impulsivity Scale (BIS) was presented in three stages of pre-test, post-test and follow-up stages. Data were analyzed using SPSS 20 software. Analysis of covariance tests were used to analyze the data.

Results

The results showed that the effect of the between-subject factor was significant for the total score (partial $\rrbracket^{2}=$ $0.32, p<0.05$ ) cognitive impulsivity (partial $\rrbracket^{2}=0.31, p<0.05$ ), motor impulsivity (partial $\rrbracket^{2}=0.18, p<0.05$ ), nonplanning (partial $\mathbb{1}^{2}=0.16, p<0.05$ ), Also, the investigation of impulsivity components also shows that there is a significant difference between combined and pharmacotherapy groups.

\section{Conclusion}

Combined treatment proved to be more effective in the treatment of patients suffering from bipolar disorder type I than separate medication. It can probably be used as a supplementary psychiatric medication, but this improvement but this improvement is not synonymous with stopping the use of psychiatric drugs.

\section{Introduction}

Bipolar disorder type I with a prevalence between 2.8 to 6.5 , is one of the most important psychiatric disorders (1). In Iran, this disorder also has different clinical features with a prevalence of $9.11 \%$ to $2.30 \%$ (2). The probability of suicide and death in these patients is high and about $15 \%$ (3). Also, the different consequences of this disorder indicate the it's mental burden imposed on the patient and caregivers (4).

Studies have showed that impulsivity plays a major role in mood disorders and is core feature of bipolar disorder $(5,6)$ which is associated with problems in cognitive functions $(7)$. Impulsivity is a multidimensional concept defined by engaging in speedy behaviors and hasty response to stimuli and often leads to uncompromising consequences (8). Impulsivity includes inhibition control and inhibition control is one of the executive functions which is a set of superior organizing and integration abilities that are related to different paths of neural interaction such as prefrontal cortex at the anatomical level. Besides, it related to some 
functions such as planning, selection. Moreover, from another view, selecting appropriate response among the various responses includes due to changing circumstances is critical function. This function is disrupted in patients with bipolar disorder type 1 completely (9).

Based on Barkleys's inhibition model (10), it is assumed that the superior functioning of executive functions depends on the perfect functioning of inhibition in the frontal and prefrontal lobes, and that response inhibition has a neurological pattern; On the other hand, impulsivity, which is one of the main elements of bipolar disorder and is prominent in all stages of the disease, is associated with response inhibition (11). Also, it seems cognitive functions and response inhibition be associated with impulsivity (7). Impulsivity is of great importance in clinical conditions because it is associated with underlying diseases, family, occupational and social problems, accidents and violence (12). Therefore, increased impulsivity can predispose individuals to develop or continuance behaviors related to more severe psychological conditions (13).

Dysfunction in performance resulting from the disease is evident during the phases of mania and depression (14). Considering this issue that less than half of bipolar patients have a favorable long-term response to treatment, and even if continued treatment, many patients do not achieve full recovery, and at the same time, many patients have low acceptance for continuous pharmacotherapy (15). Despite the effectiveness of pharmacotherapy, which is often given to patients in a controlled and accurate manner during hospitalization, the onset of symptoms and recurrence of the disorder sends patients to the hospital and creates a new cycle of the medication process (16). Although the most common treatment for these disorders is the use of antipsychotic drugs, long-term use of these drugs has a number of side effects that cause cognitive dysfunction and do not have a sufficient effect on the negative symptoms of the disease; Therefore, the development of new treatment methods seems necessary (17). Now days, one of these new treatments that is currently being considered by researchers in improving cognitive processes is the use of Transcranial Direct Current Stimuli (tDCS), which is known as a non-invasive, inexpensive and painless non-pharmacological treatment. In this method, by placing electrodes on the scalp, a continuous and direct electric current with very low intensity (maximum $2 \mathrm{~mA}$ ) enters the head and causes the membrane potential of cortical neurons to shift in the direction of depolarization or hyperpolarization. This increase or decreases the firing of neurons and, as a result, modulates their activity (18). Studies have shown that tDCS has an effect on impulsivity in psychiatric disorders $(19,20)$. Therefore, this type of treatment has had a positive effect on patients with psychiatric and neurological disorders, which shows its effectiveness. Therefore, the aim of this study was to comparison of Pharmacotherapy and combined pharmacotherapy with Transcranial Direct Current Stimuli action (tDCS) in reduction of impulsivity in patients with bipolar disorder type I.

\section{Methods}

\subsection{Procedure and study setting}

The present study is a clinical trial with pre-post-test and follow -up stages. The statistical population included all patients with bipolar I disorder in Zanjan. A sample of 30 patients with bipolar disorder was selected by purposive sampling and randomly assigned in two groups. Random allocation was done by dividing the participants into the desired groups according to the Random Number Generator.v1.3 program. The ethics committee of the Zanjan University of Medical Sciences (ZUMS) approved the clinical trial of this study 
(Approval ID: IR.ZUMS.REC.1398.452). The trial was registered at the Iranian Registry of Clinical Trials (registration No. RCTID: IRCT20191229045931N1). Samples were referred according to the inclusion criteria by a psychiatrist and if they were not fulfill the exclusion criteria, they were included in the groups. The samples were outpatients of Suhrawardi Clinic of Shahid Beheshti Hospital in Zanjan. After obtaining informed consent to participate in the study, the Bart impulsivity scale was presented. Present clinical trial was a randomized WITH parallel-group study which conducted over a 5-week period in one of the psychiatric hospitals (Shahid Beheshti Psychiatric Hospital affiliated with Zanjan University of Medical Sciences). All activities of this trial from the beginning to the end have been approved by the ethics committee of the Zanjan University of Medical Sciences (ZUMS) (Approval ID: IR.ZUMS.REC.1398.452) and its identification code in Iranian Registry of Clinical Trials is IRCT20191229045931N1. Also, its process was accordant with the Declaration of Helsinki and its principles. Patients' symptoms were measured at the baseline time and weeks fifth and follow up ( 3 months after completion of trial). To conduct the study ethically, all the steps and objectives were described to the patients. Then, a written informed consent form was given to the patients. Moreover, all necessary explanation concerning the right to withdraw the study for any reason was provided to the patients.

The tDCS protocol was performed for 10 sessions of 20 minutes (anode on F3 and cathode on F4) for the experimental group. In the treatment of direct current cranial wall stimulation, two electrodes, one positive pole and the other negative pole, are placed on the head through a sponge pad soaked in a conductive solution. The electric current by these electrodes, after passing through different areas (scalp, skull, etc.), reaches the surface of the cerebral cortex. The current that reaches this area charges the neurons with electricity and creates a positive and negative pole, which leads to a change in the activity of that area. The pharmacotherapy group also received psychiatric medication plus tDCS. It should be noted that the sample was not from the inpatient department and went to the psychiatrist's office on an outpatient basis. There was no way for groups to be as homogeneous as possible. After the intervention, the Bart impulsivity Scale (BIS) was performed again and also 3 months after the intervention in order to follow up the results.

At the end of the treatment sessions, the collected information was analyzed using multivariate methods and SPSS 20 software (IBM Corporation, Armonk, NY). In data analysis, descriptive statistics were used to classify and display the data. To test the hypotheses from covariance analysis tests (univariate and multivariate) and to check the assumptions of the mentioned test, from Levin test (for homogeneity of variances), Kolmogorov Smirnov (for data normality), for homogeneity of groups from independent t-test were used. Also, FOR testing the research hypotheses, the analysis of variance test with repeated measures was used.

\subsection{Determination of sample size}

\subsection{Participants}

Recruitment process was performed from outpatient wards (including patients 18 to 50 years of age of both sexes). All of the patients met the necessary criteria for bipolar disorder type I diagnosis according to DSM-5 (Diagnostic and Statistical Manual of Mental Disorders, Fifth Edition). Before any intervention, the psychiatrist diagnosed bipolar disorder type I based on the DSM-5 criteria for all participants and took a complete medical history. Besides, Inclusion criteria included diagnosis of bipolar disorder type I, tendency to participate in research, Age range minimum 18 and maximum 50 years old, Minimum high school education, No severe psychiatric disorders such as psychotic disorders and cognitive disorder, Lack of history of seizures and history 
of head injury, Not consuming drugs and alcohol and not receiving psychological and technological treatments at least one month before entering the research. Exclusion criteria included Absence for more than two sessions, Suicidal ideation during intervention sessions, no need to take ECT during intervention sessions, canceling continuation of intervention sessions, having a metal device or other electrical device in the head and pregnancy. Finally, 30(15 patients for every groups) patients assigned in two groups pf pharmacotherapy and combined pharmacotherapy with tDCS randomly.

\subsection{Intervention}

In order to random assignment, the sample were assigned into 2 parallel groups randomly. So, the first group only took mood stabilizers including $300 \mathrm{mg}$ lithium, $200 \mathrm{mg}$ sodium valproate, and $200 \mathrm{mg}$ carbamazepine, two times per day. Moreover, for pharmacotherapy plus TDCS group, along the previous treatment, the TDCS (2 $\mathrm{mA}$ ) was performed for 10 consecutive days, two sessions per day each for 20 minutes. To do so, two electrodes (positive Anode and negative Cathode) covered by a sponge soaked in saline were positioned in the subjects' head over dorsolateral prefrontal cortex (DLPFC) with anode position over F3 and cathode over F4 according to the EEG 10-20 International System.

\subsection{Instrument}

Barratt Impulsivity Scale: Barratt Impulsivity Scale (BIS) has 31 questions that evaluate three factors including cognitive impulsivity, motor impulsivity and nonplanning. The questions are formulated in four options and the highest score that is obtained from the sum of three factors is equal to 120 . Some authors have calculated the validity and reliability of Persian versions of the Barratt scale in determining risky behaviors and impulsive (21). Cronbach's alpha was calculated at 0.83 . Also, the subscales showed a significant correlation with each other $(\mathrm{R}=0.4)(22)$.

TDCS: Transcranial Direct Current Stimuli (TDCS) device made in Canada that has FDA approval. The protocol included stimulation over the DLPFC for 10 sessions of 20 minutes for the combined group (stimulation over left Anode/ right Cathode) [F3, F4]. During TDCS, two electrodes, one positive pole and the other negative pole, are placed on the head through a sponge pad soaked in a conductive solution. Electric current by these electrodes after passing through different areas (scalp, skull, etc.), reaches the surface of the cerebral cortex. The current that reaches this area charges the neurons with electricity and causes a positive and a negative pole, which leads to a change in the activity of that area. Depending on the specific disorder, the following should be considered: current intensity, duration and direction, location of pads, size of pads, and number of sessions.

\subsection{Safety}

All participants were assured that they were free to withdraw from the trial. Also, sufficient information was provided to inform the members of the research team in case of any unexpected side effects. Over the course of 5 weeks, there was no dropout due to different side effects.

\subsection{Data analysis}


IBM SPSS Statistic (version 20) (IBM Corporation, Armonk, NY) was applied for analysis the data. Categorical variables (number of patients and percentage) were considered and mean - SD were counted as continuous variable $(95 \%=\mathrm{Cls})$ was considered to report MD. For comparison of trend of change in Barratt Impulsivity Scale (BIS) during 5 weeks of treatments General Linear Model (GLM) repeated measure was used by analyzer. Between-subject factor and within-subject factor are counted for study groups and times of measurements, respectively. Greenhouse- Geisser correction was performed for reporting of degrees of freedom (DF) if Mauchly's test of sphericity was significant statistically. Moreover, for evaluating effectiveness of each treatment in reducing symptoms, one-way analysis of variance was performed. T-test was also used to compare of changing the status of scores from baseline in the first and second group. Two statistical methods included Fisher's exact and Chi-square for comparison categorical variables too. Also, in all stages of analysis the data a p-value $<0.05$ was considered as level of significance.

\section{Results}

Table 1. Demographic characteristics of participants in two study groups

\begin{tabular}{|c|c|c|c|c|c|c|}
\hline \multicolumn{2}{|c|}{ Demographic factors } & \multicolumn{2}{|c|}{ Medication $(n=15)$} & \multicolumn{2}{|c|}{ Medication+ tDCS $(n=15)$} & \multirow[t]{2}{*}{ P-value* } \\
\hline & & $\mathbf{N}$ & $\%$ & $\mathbf{N}$ & $\%$ & \\
\hline \multirow[t]{2}{*}{ Gender } & Male & 10 & 66.7 & 3 & 20 & \multirow[t]{2}{*}{0.01} \\
\hline & Female & 5 & 33.3 & 12 & 80 & \\
\hline \multirow[t]{2}{*}{ Marital status } & Married & 7 & 46.7 & 8 & 53.3 & \multirow[t]{2}{*}{0.71} \\
\hline & Single & 8 & 53.3 & 7 & 46.7 & \\
\hline \multirow[t]{4}{*}{ Educational level } & Lower than high school & 2 & 13 & 2 & 13.3 & \multirow[t]{3}{*}{0.90} \\
\hline & High school diploma & 9 & 60 & 10 & 66.7 & \\
\hline & Bachelor's degree & 4 & 26.7 & 3 & 20 & \\
\hline & & Mean & SD & Mean & SD & P-value** \\
\hline \multicolumn{2}{|l|}{ Age } & 30.33 & 8.63 & 32.06 & 9.43 & 0.60 \\
\hline
\end{tabular}

Table 2. Mean scores of participants in Impulsivity (pretest, posttest, follow up) 


\begin{tabular}{|c|c|c|c|}
\hline \multirow[t]{3}{*}{ Variables } & \multirow[t]{3}{*}{ Stage } & \multicolumn{2}{|l|}{ Groups } \\
\hline & & Pharmacotherapy, & Phamacotherapy+ tDCS, \\
\hline & & Mean \pm SD & Mean $\pm S D$ \\
\hline \multirow[t]{3}{*}{ Total } & Pretest & $70.40 \pm 14.19$ & $72.60 \pm 13.29$ \\
\hline & Posttest & $74 \pm 13.49$ & $66.73 \pm 14.45$ \\
\hline & Follow-up & $70.73 \pm 15.63$ & $65.86 \pm 12.52$ \\
\hline \multirow[t]{3}{*}{ Cognitive Impulsivity } & Pretest & $21.26 \pm 6.18$ & $19.26 \pm 4.21$ \\
\hline & Posttest & $22.13 \pm 3.68$ & $17.53 \pm 5.11$ \\
\hline & Follow-up & $20.46 \pm 4.59$ & $18.73 \pm 4.41$ \\
\hline \multirow[t]{3}{*}{ Motor Impulsivity } & Pretest & $22.33 \pm 5.53$ & $24.06 \pm 4.83$ \\
\hline & Posttest & $22.60 \pm 5.75$ & $22.33 \pm 5.19$ \\
\hline & Follow-up & $23.26 \pm 6.52$ & $21.0 \pm 4.95$ \\
\hline \multirow[t]{3}{*}{ nonplanning } & Pretest & $27.26 \pm 5.48$ & $29.53 \pm 6.39$ \\
\hline & Posttest & $27.93 \pm 6.27$ & $27.6 \pm 5.79$ \\
\hline & Follow-up & $27.20 \pm 6.38$ & $25.66 \pm 5.71$ \\
\hline
\end{tabular}

Table 3. The results of MANCOVA on Impulsivity 


\begin{tabular}{|lllllll|}
\hline Source & Sum of Squares & df & Mean Square & F & Sig. & Partial Eta squared \\
\hline Total corrected model & 3417.32 & 5 & 683.46 & 6.69 & 0.000 & 0.58 \\
\hline Cognitive impulsivity & 332.71 & 5 & 6654 & 4.18 & 0.007 & 0.46 \\
\hline Motor impulsivity & 520.74 & 5 & 104.14 & 7.52 & 0.000 & 0.61 \\
\hline Nonplanning & 54162 & 5 & 108.32 & 5.25 & 0.002 & 0.52 \\
\hline Total Intercept & 225.19 & 1 & 225.19 & 3.18 & 0.087 & 0.11 \\
\hline Cognitive impulsivity & 49.91 & 1 & 49.91 & 3.14 & 0.089 & 0.11 \\
\hline Motor impulsivity & 19.25 & 1 & 19.25 & 1.29 & 0.250 & 0.05 \\
\hline Nonplanning & 60.98 & 1 & 60.98 & 3.01 & 0.095 & 0.11 \\
\hline Total pretest & 618.86 & 1 & 618.86 & 6.21 & 0.02 & 0.20 \\
\hline Cognitive impulsivity & 2.47 & 1 & 2.47 & 0.15 & 0.69 & 0.00 \\
\hline Motor impulsivity & 41.58 & 1 & 41.58 & 2.80 & 0.10 & 0.10 \\
\hline Nonplanning & 260.77 & 1 & 260.77 & 13.01 & 0.000 & 0.35 \\
\hline Total Group & 1134.78 & 1 & 1134.78 & 11.38 & 0.000 & 0.32 \\
\hline Cognitive impulsivity & 172.41 & 1 & 173.41 & 10.91 & 0.000 & 0.31 \\
\hline Motor impulsivity & 78.02 & 1 & 78.02 & 5.26 & 0.03 & 0.18 \\
\hline Nonplanning & 95.18 & 1 & 95.18 & 4.75 & 0.03 & 0.16 \\
\hline Total Error & 245.64 & 24 & 102.15 & & & \\
\hline Cognitive impulsivity & 381.45 & 24 & 15.89 & & & \\
\hline Motor impulsivity & 332.22 & 24 & 13.84 & & & \\
\hline Nonplanning & 485.88 & 24 & 20.24 & & & \\
\hline Total & 154413 & 30 & & & & \\
\hline Cognitive impulsivity & 12515 & 30 & & & & \\
\hline Motor impulsivity & 16677 & 30 & & & & \\
\hline Nonplanning & 23715 & 30 & & & & \\
\hline
\end{tabular}

Table 4. Test of repeated measure on impulsivity 


\begin{tabular}{|lllllll|}
\hline Source & $\begin{array}{l}\text { Sum of } \\
\text { Squares }\end{array}$ & df & $\begin{array}{l}\text { Mean } \\
\text { Square }\end{array}$ & F & Sig. & $\begin{array}{l}\text { Partial Eta } \\
\text { squared }\end{array}$ \\
\hline Total score & 318.28 & 2 & 159.14 & 2.71 & 0.07 & 0.08 \\
\hline Total score.group & 336.15 & 2 & 168.07 & 2.87 & 0.06 & 0.09 \\
\hline Cognitive impulsivity & 95.75 & 1.41 & 42.26 & 1.40 & 0.25 & .04 \\
\hline $\begin{array}{l}\text { Cognitive } \\
\text { impulsisity.group }\end{array}$ & 92.06 & 1.41 & 65.12 & 2.16 & 0.14 & 0.07 \\
\hline Motor impulsivity & 18.86 & 2 & 9.43 & .6 & 0.35 & 0.35 \\
\hline Motor impulsivity.group & 65.00 & 2 & 35.50 & 3.67 & 0.03 & 0.11 \\
\hline Nonplanning & 58.15 & 2 & 29.7 & 3.12 & 0.05 & 0.100 \\
\hline $\begin{array}{l}\text { Nonplanning } \\
\text { Group }\end{array}$ & 61.75 & 2 & 30.87 & 3.32 & 004 & 0.14 \\
\hline
\end{tabular}

Examination of the results of repeated measurements shows that in this analysis only the interactive effect of the group on the time levels of motor impulsivity variables $(F=3.12)$ and nonplanning $(F=3.67)$ are significant.

Table 5. Pairwise comparsion for the impulsivity variable

\begin{tabular}{|lllllll|}
\hline $\begin{array}{l}\text { Dependent } \\
\text { variable }\end{array}$ & groups & & Mean & $\begin{array}{l}\text { Mean } \\
\text { differences }\end{array}$ & $\begin{array}{l}\text { Std. } \\
\text { error }\end{array}$ & Sig. \\
\hline Total score & tDCS+pharmacotherapy & pharmacotherapy & 77.25 & -13.78 & 4.08 & 0.00 \\
\hline & pharmacotherapy & tDCS+pharmacotherapy & 63.47 & 13.78 & 4.8 & 0.00 \\
\hline $\begin{array}{l}\text { Cognitive } \\
\text { impulsivity }\end{array}$ & $\begin{array}{l}\text { tDCS } \\
\text { tpharmacotherapy }\end{array}$ & pharmacotherapy & 22.52 & -5.38 & 1.63 & 0.00 \\
\hline & pharmacotherapy & tDCS+pharmacotherapy & 17.13 & 5.38 & 1.63 & 0.00 \\
\hline $\begin{array}{l}\text { Motor } \\
\text { impulsivity }\end{array}$ & $\begin{array}{l}\text { tDCS } \\
\text { tpharmacotherapy }\end{array}$ & pharmacotherapy & 24.77 & -3.61 & 1.57 & 0.03 \\
\hline & pharmacotherapy & tDCS+pharmacotherapy & 21.15 & 3.61 & 1.57 & 0.03 \\
\hline Nonplanning & tDCS+pharmacotherapy & pharmacotherapy & 29.49 & -3.99 & 1.83 & 0.03 \\
\hline & pharmacotherapy & tDCS+pharmacotherapy & 25.50 & 3.99 & 1.3 & 0.03 \\
\hline
\end{tabular}

As can be seen in the table above, there is a significant difference between the total score as well as all the impulsivity subscales in the group. The investigating of the means of the two groups also shows that the means of the combined group is lower than the pharmacotherapy group. That is, the mean impulsivity in people in the combined group was significantly reduced compared to the Pharmacotherapy group. 


\section{Discussion}

The aim of this study was to compare the effectiveness of pharmacotherapy and combined pharmacotherapy with Transcranial Direct Current Stimuli action (tDCS) in reduction of impulsivity in patients with bipolar disorder type I. Analysis of the findings indicates the effectiveness of combined pharmacotherapy and tDCS with in reducing impulsivity is stable. Bipolar disorder is associated with high impulsivity and this leads to a decline in social functions (23). Therefore, research has been done in line with the results of the present study. Mayer et al., For example, concluded in a review study that TDCS can reduce impulsivity in a healthy general population as well as in patients with psychiatric disorders, and is therefore consistent with our research findings (20). Georgii also concluded that by stimulating the right DLPFC, impulsivity is reduced and purposeful and planned behaviors increased in individuals (24). Besides, other research is in line with our research findings $(19,25,26)$.

In more detail explaining the finding that TDCS can reduce impulsivity, it can be said that the frontal cortex plays an important role in cognitive control (27), which determines the goals and how to achieve it, as well as basic functions such as inhibitory control, attention, planning and risk-behaviors. ow activity of the prefrontal cortex may lead to defects in these functions, leading to increased cognitive and motor impulsivity as well as unplanned activities; Therefore, inhibition control prevents automatic responses and stops cognitive processes or inappropriate actions that may interfere with the proper performance of the intended action. As a result, inhibition control acts as a filter and is associated with social adequacy and behavioral and emotional control, and impaired inhibition control causes impulsivity (28).

From another perspective, high impulsivity seems to indicate an imbalance between reward-sensitive areas and areas involved in cognitive control, so impulsive behavior may be due to incoherence between reward areas and cognitive control (29). As a result, the use of tDCS in the frontal areas improves the reward path through direct top-down processes and indirectly by improving cognitive processes such as impulsivity. Therefore, the use of tDCS on DLPFC in patients with bipolar disorder can improve their cognitive function by reducing risk-taking and impulsivity behavior.

Explaining the mechanism of action of this type of treatment, it can be said that the usual protocols for using tDCS are through two electrodes attached to the skin, one as the anode and the other as the cathode. An electric current of one to two milliamperes is applied for twenty minutes between these two electrodes, each of which usually has a cross-sectional area of 35 square centimeters. The direction of current is from the anode to the cathode and according to the direction and intensity of the current, the amount of excitability of the cerebral cortex increases or decreases (30). tDCS Anode has a stimulating effect on neurons by depolarizing neurons, while its cathodic type inhibits neuronal tissue by hyperpolarizing neurons (31). Overall, the use of tDCS protocols has shown that this method has no significant side effects and only causes mild headaches and itching under the electrodes. According to the data, tDCS does not generate heat, does not cause neuronal damage, cerebral edema and changes in the blood-brain barrier, and has no adverse effects during the treatment of depressed patients (32).

Pharmacological studies have been instrumental in answering how tDCS works. For example, sodium and calcium channel blockers can prevent the immediate and long-term effects of Anodic stimulation, and NMDA glutamate blockers prevent the long-term effects of tDCS, regardless of stimulation (31). Cathode tDCS 
mediates long-term effects on spontaneous activity in peripheral and central nervous tissue by affecting mainly the membrane potential of neurons rather than the strength and activity of synapses (33). These results mean that tDCS has the ability to alter the excitability of excitatory neurons as well as interneurons. As a result, tDCS appears to have a set of depolarizing, hyperpolarizing effects on axonal activity and also affects synaptic function (33).

On the other hand, the dorsal-lateral frontal cortex because it plays an important role in cognitive functions, especially in inhibitory concentration and control (30). Targeting this cortex of the brain seems to have a high capacity to promote cognitive function and interfere with pathological behaviors (34). In general, TDCS can regulate brain activity in a way that is directly related to function (35) and it has long-term effects that can be used for patients who show side effects of drugs and are resistant to medical symptoms (36).

\section{Conclusion}

Bipolar disorder is a group of psychiatric disorders that, due to its strong biological nature, requires complementary therapies and brain rehabilitation, and in most cases, the use of drugs alone cannot help improve the cognitive, behavioral and social functions of these people. Therefore, conducting clinical trials and, consequently, evaluating the effectiveness of biologic therapies can help improve the function of these patients. In this study, the combination of TDCS and drug therapy, compared to drug therapy alone, could significantly improve impulsivity reduction and could possibly be used as a complementary therapy, in addition to the use of psychiatric drugs.

Some limitations of the present study included: low sample size, lack of review of different tDCS protocols. Another limitation is the low sample size, which reduces the generalizability of the findings. Therefore, it is suggested that future research be used in larger sample sizes, using objective tools such as EEG, fMRI. It is also suggested that the treatment based on transcranial stimulation of the brain be compared with other modern therapies such as neurofeedback, rTMS or psychotherapy.

\section{Declarations}

\section{Ethics approval and consent to participate}

All activities of this trial from the beginning to the end have been approved by the ethics committee of the Zanjan University of Medical Sciences. Approval ID: IR.ZUMS.REC.1398.452

\section{Consent for publication}

All authors contributed to and approved the final manuscript and gave their consent for publication

\section{Availability of data and materials}

The datasets used during the current study are available from the corresponding author on reasonable request.

\section{Competing interests}

The authors declare that they have no competing interests. 


\section{Funding/Support}

Not applicable.

\section{Authors' contributions}

All authors made substantial contributions to the conception or design of the work, or the acquisition, analysis or interpretation of data. Authors SEM and MD designed the experiment. Authors PD, AZ and HJ acquired the data. Authors SEM, PM and MD analyzed the data. Authors SEM and PM undertook the literature searches and wrote the first draft of the manuscript. All authors contributed to and revised the manuscript. All authors read and approved the final manuscript.

\section{Acknowledgments}

The authors would like to thank the staff of health information department in ZUMS for providing treat the patients during three-month period. Finally, the authors appreciate the permission and guidance that the department for expanding clinical research in ZUMS provided.

\section{References}

1. Bauer M, Pfennig A. Epidemiology of bipolar disorders. Epilepsia. 2005;46:8-13.

2. Mohammadi M, Rahgozar M, Bagheri Yazdi A, Naghavi H, Pour Etemad HR, Amini H, et al. Epidemiological study of psychiatric disorders in Tehran province. Iranian Journal of Psychiatry and Clinical Psychology. 2003;9(2):4-13.

3. Vieta E, Benabarre A, Colom F, Gastó C, Nieto E, Otero A, et al. Suicidal behavior in bipolar I and bipolar II disorder. The Journal of nervous and mental disease. 1997;185(6):407-9.

4. McMorris BJ, Downs KE, Panish JM, Dirani R. Workplace productivity, employment issues, and resource utilization in patients with bipolar I disorder. Journal of medical economics. 2010;13(1):23-32.

5. Omar A, Elbatrawy A, Sabry W, Elkholy H, Farrag W. Impulsivity as a Clinical Marker for Bipolar Affective Disorder. QJM: An International Journal of Medicine. 2020;113(Supplement_1):hcaa054. 28.

6. McDonald CE, Rossell SL, Phillipou A. The comorbidity of eating disorders in bipolar disorder and associated clinical correlates characterised by emotion dysregulation and impulsivity: A systematic review. Journal of affective disorders. 2019;259:228-43.

7. Jiménez E, Arias B, Castellví P, Goikolea JM, Rosa A, Fananas L, et al. Impulsivity and functional impairment in bipolar disorder. Journal of affective disorders. 2012;136(3):491-7.

8. Moeller FG, Barratt ES, Dougherty DM, Schmitz JM, Swann AC. Psychiatric aspects of impulsivity. American journal of psychiatry. 2001;158(11):1783-93.

9. Lezak MD, Howieson DB, Loring DW, Fischer JS. Neuropsychological assessment: Oxford University Press, USA; 2004.

10. Barkley RA, Biederman J. Toward a broader definition of the age-of-onset criterion for attention-deficit hyperactivity disorder. Journal of the American Academy of Child \& Adolescent Psychiatry. 1997;36(9):1204-10. 
11. Ekinci O, Albayrak Y, Ekinci AE, Caykoylu A. Relationship of trait impulsivity with clinical presentation in euthymic bipolar disorder patients. Psychiatry Research. 2011;190(2-3):259-64.

12. Najt P, Perez J, Sanches M, Peluso M, Glahn D, Soares JC. Impulsivity and bipolar disorder. European neuropsychopharmacology. 2007;17(5):313-20.

13. Arianakia E, Hasani J. Impulsivity and cognitive emotion regulation strategies in patients with bipolar-and major depressive disorders. Advances in Cognitive Science. 2014;16(2):1-10.

14. Vojta C, Kinosian B, Glick H, Altshuler L, Bauer MS. Self-reported quality of life across mood states in bipolar disorder. Comprehensive psychiatry. 2001;42(3):190-5.

15. Akiskal SH. Sadock BJ SV, Ruiz P. Kaplan and Sadock's comprehensive textbook of psychiatry. 9th ed. Philadelphia: Lippincott Williams and Wilkins;2009.

16. Harrow M, Goldberg JF, Grossman LS, Meltzer HY. Outcome in manic disorders: a naturalistic follow-up study. Archives of General Psychiatry. 1990;47(7):665-71.

17. Mohammadi A, Hesami E, Kargar M, Shams J. Detecting allocentric and egocentric navigation deficits in patients with schizophrenia and bipolar disorder using virtual reality. Neuropsychological rehabilitation. 2018;28(3):398-415.

18. Arkan A. Effect of transcranial direct current stimulation (tDCS) on working memory in healthy people. Brain Stimulation: Basic, Translational, and Clinical Research in Neuromodulation. 2019;12(2):385.

19. Teti JM, Nicolier M, Gabriel D, Masse C, Giustiniani J, Compagne C, et al. Efficacy of transcranial direct current stimulation in reducing impulsivity in borderline personality disorder (TIMBER): study protocol of a randomized controlled clinical trial. Trials. 2019;20(1):347-.

20. Mayer JT, Chopard G, Nicolier M, Gabriel D, Masse C, Giustiniani J, et al. Can transcranial direct current stimulation (tDCS) improve impulsivity in healthy and psychiatric adult populations? A systematic review. Progress in Neuro-Psychopharmacology and Biological Psychiatry. 2020;98:109814.

21. Ekhtiari H SH, Esmaeeli Djavid G, Atefvahid M, Edalati H, Mokri A. Reliability and Validity of Persian Versions of Eysenck, Barratt, Dickman and Zuckerman Questionnaires in Assessing Risky and Impulsive Behaviors. Iranian Journal of Psychiatry and Clinical Psychology. 2008;14(3):326-36.

22. Taremian F, Nazari S, Moradveisi L, Moloodi R. Transcranial direct current stimulation on opium craving, depression, and anxiety: a preliminary study. The journal of ECT. 2019;35(3):201-6.

23. Ozten M, Erol A. Impulsivity differences between bipolar and unipolar depression. Indian journal of psychiatry. 2019;61(2):156.

24. Georgii C, Goldhofer P, Meule A, Richard A, Blechert J. Food craving, food choice and consumption: The role of impulsivity and sham-controlled tDCS stimulation of the right dIPFC. Physiology \& behavior. 2017;177:20-6.

25. Allenby C, Falcone M, Bernardo L, Wileyto EP, Rostain A, Ramsay JR, et al. Transcranial direct current brain stimulation decreases impulsivity in ADHD. Brain stimulation. 2018;11(5):974-81.

26. Bell SB, Turner B, Sawaki L, DeWall N. When brain stimulation backfires: the effects of prefrontal cortex stimulation on impulsivity. Social cognitive and affective neuroscience. 2020;0(0):1-8.

27. Moslemi B, Azmodeh M, Tabatabaei M, Alivandi Vafa M. The Effect of Transcranial Direct Current Stimulation on Dorsolateral Prefrontal Cortex: a Review of its Role on Cognitive Functions. The 
Neuroscience Journal of Shefaye Khatam. 2019;8(1):129-44.

28. Moradi K YS, Abdolahi F, Hossen M. Effectiveness of transcranial direct current stimulation on dorsolateral prefrontal cortex in cigarette craving and stress. Journal OF Research in Psychological health. 2016;10(3):7-30.

29. Gorini A, Lucchiari C, Russell-Edu W, Pravettoni G. Modulation of risky choices in recently abstinent dependent cocaine users: a transcranial direct-current stimulation study. Frontiers in human neuroscience. 2014;8:661.

30. Miranda PC, Lomarev M, Hallett M. Modeling the current distribution during transcranial direct current stimulation. Clinical neurophysiology. 2006;117(7):1623-9.

31. Nitsche MA, Fricke K, Henschke U, Schlitterlau A, Liebetanz D, Lang N, et al. Pharmacological modulation of cortical excitability shifts induced by transcranial direct current stimulation in humans. The Journal of physiology. 2003;553(1):293-301.

32. Nitsche M, Niehaus L, Hoffmann K, Hengst S, Liebetanz D, Paulus W, et al. MRI study of human brain exposed to weak direct current stimulation of the frontal cortex. Clinical Neurophysiology. 2004;115(10):2419-23.

33. Ardolino G, Bossi B, Barbieri S, Priori A. Non-synaptic mechanisms underlie the after-effects of cathodal transcutaneous direct current stimulation of the human brain. The Journal of physiology. 2005;568(2):65363.

34. Eslamizade MJ, Behbahanian S, Mahdavi SM, Oftadehal M. An introduction to neurotechnologies, transcranial magnetic stimulation and transcranial direct current stimulation: Their applications in the cognitive enhancement and rehabilitation. The Neuroscience Journal of Shefaye Khatam. 2016;4(2):65-86.

35. Giordano J, Bikson M, Kappenman ES, Clark VP, Coslett HB, Hamblin MR, et al. Mechanisms and effects of transcranial direct current stimulation. Dose-Response. 2017;15(1):1559325816685467.

36. Eskandari Z, Dadashi M, Mostafavi H, Kia AA, Pirzeh R. Comparing the efficacy of anodal, cathodal, and sham transcranial direct current stimulation on brain-derived neurotrophic factor and psychological symptoms in opioid-addicted patients. Basic and Clinical Neuroscience. 2019;10(6):641.

\section{Supplementary Files}

This is a list of supplementary files associated with this preprint. Click to download.

- 1.jpg 